\title{
Correction to: Combined gene expression and proteomic analysis of EGF induced apoptosis in A431 cells suggests multiple pathways trigger apoptosis
}

\author{
Ibrahim Alanazi $^{1,2} \cdot$ Esmaeil Ebrahimie $^{1} \cdot$ Peter Hoffmann $^{1} \cdot$ David L. Adelson $^{1}$
}

Published online: 12 December 2017

๑) Springer Science+Business Media, LLC, part of Springer Nature 2017

\section{Correction to: Apoptosis (2013) 18:1291-1305 https://doi.org/10.1007/s10495-013-0887-6}

The original version of this article unfortunately contained a mistake. The affiliation of first author Dr. Ibrahim Alanazi was incorrect.

The first author inadvertently missed to include his second affiliation in the original article. The correct affiliation for Dr. Ibrahim Alanazi is "School of Molecular \& Biomedical Science, The University of Adelaide, Adelaide, Australia" and "National Centre for Genomics, King Abdulaziz City for Science and Technology (KACST), P.O. Box 6086, Riyadh 11442, Saudi Arabia”.

The online version of the original article can be found under https://doi.org/10.1007/s10495-013-0887-6.

David L. Adelson

david.adelson@adelaide.edu.au

1 School of Molecular \& Biomedical Science, The University of Adelaide, Adelaide, SA, Australia

2 National Centre for Genomics, King Abdulaziz City for Science and Technology (KACST), P.O. Box 6086, Riyadh 11442, Saudi Arabia 